\title{
AN UNNECESSARY CONDITION IN TWO THEOREMS OF ANALYSIS SITUS*
}

BY P. M. SWINGLE

1. Introduction. In this paper $K$ will be a closed point set such that, if $S$ refers to the space under consideration, $S-K=S_{1}+S_{2}$. One or more of the following conditions will hold.

Condition 1. Every simple continuous arc which joins a point of $S_{1}$ to a point of $S_{2}$ contains at least one point of $K$.

Condition 2. Every point $P$ of $S_{i}(i=1$, and 2) can be joined to any point $Q$ of $K$ by a simple continuous arc $P Q$ such that $P Q-Q$ is contained in $S_{i}$.

Condition 3. Every two points of $S_{i}(i=1$, and 2$)$ can be joined by a simple continuous arc contained in $S_{i}$.

Schoenflies, $\uparrow$ using the definition of a simple closed curve given by Jordan, stated and proved the following theorem for a plane $S$.

Theorem A. If $K$ is bounded and if Conditions 1, 2, and 3 hold, then $K$ is a simple closed curve.

This theorem was later proved by Lennesł, using his own definition of a simple closed curve. In his paper On the foundations of plane analysis situs, R. L. Moore $\S$ points out that

* Presented to the Society, December 29, 1925.

$\dagger$ A. Schoenflies, Ueber einen grundlegenden Satz der Analysis Situs, Göttinger Nachrichten, 1902, p. 185.

$\ddagger N$. J. Lennes, Curves in non-wetrical analysis situs with an application in the calculus of variations, A.merican Journal, vol. 33 (1911), pp. 287-326, Lennes defines a simple continuous arc from $A$ to $B$ as a closed, bounded, connected foint set containing $A+B$ but containing no proper connected subset that contains $A+B$. He defines a simple closed curve as the sum of two simple continuous arcs which have their end points and only their end points in common.

$\S$ Transactions of this Society, vol. 17 (1916), p. 131-164, Theorem 48. This paper will be referred to hereafter as $F$. A. 
this theorem is true in a space $S$ satisfying his set of Axioms $\Sigma_{3}$. J. R. Kline* stated and proved the following theorem for a space $S$ satisfying $\Sigma_{3}$.

Theorem B. If $K$ is such that Conditions 1, 2, and 3 hold, and if $S_{i}(i=1$, and 2$)$ is unbounded, then $K$ is an open curve.†

In this paper it is proposed to show that Condition 3 is unnecessary in both Theorem A and Theorem B. The proof will be given on the basis of R. L. Moore's set of Axioms $\Sigma_{3}$. The letter $S$ will be used to represent a space satisfying these axioms. Proofs of these theorems will first be given, using only Conditions 1 and 2, which for Theorem A is independent both of the proof by Schoenflies and of that by Lennes and which for Theorem $B$ is independent of that by Kline. It will then be shown in a very simple manner, independent of these proofs, that Condition 3 is unnecessary in the presence of the other two conditions. I wish to thank Professor R. L. Wilder for his valuable sugge stions.

2. Definitions and Proofs.

Definition A. $\ddagger A$ simple continuous arc from $A$ to $B$ ( $A$ and $B$ distinct points) is a closed, bounded, $\S$ connected point set, $M$, containing $A+B$ such that if $X$ is any point of $M$ distinct from $A$ and $B$, then $M-X$ is disconnected.

* Transactions of this Society, vol. 18 (1917), p. 177-184. Lennes' definition of a simple continuous arc is used.

$\dagger$ An open curve is a closed, connected set of points, $M$, such that if $P$ is a point of $M$, then $M-P$ is disconnected. A point set is closed if it contains all of its own limit points. A set $N$ is disconnected (or not connected) if there exist point sets $U$ and $V$ such that $N=U+V$ separate. A point set which cannot be so divided is connected. By $N=U+V$ separate is meant that $N$ is the sum of two non-vacuous mutually exclusive point sets $U$ and $V$ such that neither contains a limit point of the other.

$\ddagger$ R. L. Moore, Concerning simple continuous curves, Transactions of this Society, vol. 21 (1920), pp. 313-320, Def. 2.

$\S$ A point set $M$ is bounded if there exists a finite set of regions $R_{1}$, $R_{2}, \cdots, R_{n}$ such that $M$ is contained in $R_{1}{ }^{\prime}+R_{2}{ }^{\prime}+\cdots+R_{n}{ }^{\prime}$, where $R_{i}^{\prime}=R_{i}+\left(\right.$ the limit points of $R_{i}$ which are not contained in $R_{i}$ ). 
Definition B.* If $A X B$ and $A Y B$ are two simple continuous arcs having only $A$ and $B$ in common, then the point set composed of these two arcs is a simple closed (Jordan) curve.

Definition C. $\dagger$ If $M$ is a closed, connected point set which lies in a space $S$ and consists entirely of non-cut points, but which is disconnected by the omission of any two of its points, then $M$ is a simple closed (Jordan) curve.

Definition. A region $Z$ will be a region bounded by a simple closed curve $C$ such that $C \times K \ddagger=A_{1}+A_{2}$, where $A_{1}$ and $A_{2}$ are any two points of $K$, where $C=A_{1} X_{1} A_{2}+A_{2} X_{2} A_{1}$, and where $A_{1} X_{i} A_{2}(i=1,2)$ is an arc contained in $S_{i}+\left(A_{1}+A_{2}\right)$; and $K_{1}$ will represent $K \times Z$ and $K_{2}$ will represent $K \times\left(S-Z^{\prime}\right) . \S$

Theorems 1-47. These theorems are given in F. A.

Lemma I. If $K$ is such that Condition 1 holds, then there exist at least two distinct points of $K$.

Since $S$ is connected, by Theorem 22 , and is then a domain, there exists a simple continuous arc, by Theorem 15 , joining a point of $S_{1}$ to a point of $S_{2}$. This arc contains at least one point of $K$, by Condition 1 . Let $N$ be a region containing this point such that $S-N^{\prime}$ contains a point $P$ of $S_{1}$ and a point $Q$ of $S_{2}$. Since $N^{\prime}$ is a closed point set and since $S-N^{\prime}$ is connected, by Axiom $3, S-N^{\prime}$ is a domain and there exists an arc $P Q$ contained in $S-N^{\prime}$, by Theorem 15. This arc contains at least one point of $K$, by Condition 1 . Therefore there exist at least two distinct points of $K$.

*N. J. Lennes, loc. cit.

$\dagger$ This definition is due to J. R. Kline, this Bulletin, vol. 27 (1921), p. 399. R. L. Wilder has proved that this definition is equivalent to Definition B in our space $S$; see this Bulletin, vol. 31 (1925), p. 224.

$\ddagger$ If $C$ and $K$ are point sets $C \times K$ is the point set common to $C$ and $K$.

$\S$ If $N$ is a point set, $N^{\prime}$ will represent the point set composed of $N$ and its limit points. 
Lemma II. If $K$ is such that Conditions 1 and 2 hold, and if $A_{1}$ and $A_{2}$ are any two points of $K$, then there exists a region $Z$, and $A_{i}(i=1$, and 2$)$ is a limit point of both $K_{1}$ and $K_{2}$.

Let $W$ be a point of $S_{1}$. There exists an $\operatorname{arc} A_{1} W$, by Condition 2. such that $A_{1} W-A_{1}$ is contained in $S_{1}$ and an arc $A_{2} W$ such that $A_{2} W-A_{2}$ is contained in $S_{1}$. There exists then an arc $A_{1} Y_{1} A_{2}{ }^{*}$ such that $A_{1} Y_{1} A_{2}-\left(A_{1}+A_{2}\right)$ is contained in $S_{1}$. Similarly there exists an arc $A_{1} Y_{2} A_{2}$ such that $A_{1} Y_{2} A_{2}-\left(A_{1}+A_{2}\right)$ is contained in $S_{2}$. Let $C$ be the simple closed curve $A_{1} Y_{1} A_{2}+A_{2} Y_{2} A_{1}$. The region bounded by $C$ is then a region $Z$.

In any region, $L$, containing $A_{i}(i=1$, or 2$)$ there exists by Theorem 28 an $\operatorname{arc} W_{1} B_{1} W_{2}$ contained in $Z$ (or in $S-Z^{\prime}$ ) such that $W_{j}(j=1$, or 2$)$ is contained in $S_{j}$. This arc contains at least one point of $K$ by Condition 1. Therefore $A_{i}$ is a limit point of $K_{1}$ (or of $K_{2}$ ).

Lemma III. If there exists a region $Z$, and if $K_{1}^{\prime}=K_{11}+K_{12}$ separate, then there exists a simple closed curve $J_{1 i}(i=1$, and 2) enclosing points of $K_{1 i}$ and containing at most $A_{1}+A_{2}$ of $K$ and such that $J_{11} \times J_{12}=0$ and $J_{1 i}$ is contained in $Z^{\prime}$.

Since $K_{1}+\left(A_{1}+A_{2}\right)=K_{1}^{\prime}$ is closed and $K_{11}$ and $K_{12}$ are separate, $K_{1 i}(i=1$, and 2) is closed. Let $P$ be any point of $K_{11}$, except $A_{1}$ and $A_{2} . \quad P$ is not a limit point of $K_{12}$ for $K_{12}$ and $K_{11}$ are mutually exclusive closed point sets. There then exists a region, $W$ say, containing $P$ but not containing a point of $K_{12}$. Then there exists a region, $Y$ say, contained in $Z \times W$, which contains $P$. Therefore there exists a region, $L$, by Theorem 5 , which contains $P$ and is such that $L^{\prime}$ is contained in $Y$. Therefore $L^{\prime} \times K_{12}=0$. Thus for each point $P$ of $K_{11}-\left(A_{1}+A_{2}\right)$ there exists a region $L$ containing $P$ such that $L^{\prime}$ is contained in $Z$ and does not contain a point of $K_{12}$.

* Use is here made of the fact: if $A B$ is an arc and $K$ is a closed point set having at least one point in common with $A B$, then there exists on $A B$ a voint belonging to $K$ which precedes every other point of $K$ on $A B$. 
If $A_{j}(j=1$, or 2$)$ is contained in $K_{11}$ there exists a region, $Q_{j}$ say, containing the one $A_{j}$ but not the other and such that $Q_{i}{ }^{\prime} \times K_{12}=0$. Then there exists an arc $B_{j 1} X_{j} B_{j 2}$ contained in $Z^{\prime}$, by Theorem 28 , such that it has $B_{j 1}(j=1$, and 2$)$ and only these points common with $C$, and such that this arc and the $\operatorname{arc} B_{j 1} A_{j} \cdot B_{j 2}$ of $C$ is contained in $Q_{j}$. Let $T_{j 1}$ be the region bounded by $B_{j 1} X_{j} B_{j 2}+B_{j 2} A_{j} B_{j 1}$. It is contained in $Z$, by Theorem 21. Similarly there exists an arc $C_{j 1} Y_{j} C_{j 2}$ contained in $S-Z$, by Theorem 28 , but otherwise having the properties of the above arc. Let $T_{j 2}$ be the region bounded" by $C_{j 1} Y_{j} C_{j 2}+C_{j 2} A_{j} C_{j 1}$. It is contained in $S-Z^{\prime}$. Take now for example, the case where $C_{j 1} A_{j} C_{j 2}$ contains $B_{j 1} A_{i} B_{j 2}$. There exists then, by Theorem 27 , a region $T_{j}=T_{j 1}+T_{j 2}$ $+\left(B_{j 1} A_{j} B_{j 2}-\left(B_{j 1}+B_{j 2}\right)\right)$. The set $T_{i}^{\prime}$ does not contain points of $K_{12}$.

Since $K_{11}$ is bounded and closed it has the Heine-Borel property, ${ }^{*}$ by Theorem 12 . There exists then a finite number of regions, $L_{1}, L_{2}, \cdots, L_{n}$ say, of the set $(L)+\left(T_{i}\right)$ containing all the points of $K_{11}$. All the above regions can be taken with simple closed curves as boundaries, by Theorem 36 . There then exists a subset, $G$ say, of $L_{1}+L_{2}+\cdots+L_{n}$ such that each region of $G$ has a simple closed curve as boundary and such that their sum forms a maximal connected subset of $L_{1}+L_{2}+\cdots+L_{n}$. Replace $T_{j}$ by $T_{j 1}$ in case $T_{j}$ is a region of $G$. There exists by Theorem 42 a simple closed curve, $J_{11}$, which is a subset of the boundaries of the regions of $G$ and which encloses $G$. The curve $J_{11}$ contains at most $A_{1}+A_{2}$ of $K$, for only these points of $K$ are on the boundaries of $(L)$ and $T_{j 1}$ and not contained in a region of $(L)$.

Since $K_{12}$ and $L_{1}{ }^{\prime}+L_{2}{ }^{\prime}+\cdots+L_{n}{ }^{\prime}$ are mutually exclusive closed point sets, there exists a region, $M$, for each point, except $A_{1}$ and $A_{2}$, of $K_{12}$ such that $M^{\prime}$ is contained in $Z$ and such that it does not contain points of $L_{1}{ }^{\prime}+L_{2}{ }^{\prime}+\cdots$ $+L_{n}{ }^{\prime}$. If $A_{i}(i=1$, or 2$)$ is contained in $K_{12}$, take $Q_{i}$ such that

* A point set is said to have the Heine-Borel property if it is true that for every infinite collection $H$ of regions covering it, there exists a finite collection of regions belonging to $H$ and covering it. 
$Q_{i}{ }^{\prime}$ does not contain points of $L_{1}{ }^{\prime}+L_{2}{ }^{\prime}+\cdots+L_{n}{ }^{\prime}$ and so obtain $T_{i}$ as above. There then exists a simple closed curve $J_{12}$ enclosing a maximal connected subset, $H$ say, of regions of the set $(M)+\left(T_{i 1}\right)$, where $H$ is composed of a finite number of regions, and $J_{12}$ is a subset of the boundaries of the regions of $H$.

$J_{12}$ contains at most $A_{1}+A_{2}$ of $K$ and $J_{11} \times J_{12}=0$.

Lemma IV. If there exists a region $Z$, if $K_{2}{ }^{\prime}$ is a bounded point set, and if $K_{2}{ }^{\prime}=K_{21}+K_{22}$ separate, then there exists a simple closed curve $J_{2 i}\left(i=1\right.$, and 2) enclosing points of $K_{2 i}$, containing at most $A_{1}+A_{2}$ of $K$, and such that $J_{2 i}$ is contained in $S-Z$ and $J_{21} \times J_{22}=0$.

The proof is the same as in Lemma III.

Lemma V. If $K$ is such that Condition 1 holds, and if $J$ is a simple closed curve contaning at most one point, $A$ say, of $K$, then $J-A$ is contained either in $S_{1}$ or in $S_{2}$.

Assume that $J$ contains a point $B_{i}(i=1,2)$ of $S_{i}$. Then $J$ is the sum of two arcs, $B_{1} A B_{2}$ and $B_{1} B_{2}$. Each of these arcs contains at least one point of $K$, by Condition 1 , and therefore $J$ contains at least two distinct points of $K$, contrary to hypothesis.

Lemma VI. If $K$ is such that Condition 2 holds, if $J$ is a simple closed curve contained in $S_{c}(c=1$, or 2$)$ except for the point set $(A)=J \times K$ which is either vacuous or contains but one point, $A$, and if $J$ encloses at least one point of $K$, then $J$ encloses $K-(A)$ and $S_{i}(i=2$, or 1$)$.

Let $R$ be the region enclosed by $J$. Say $S_{c}=S_{1}$. Assume that there exists a point, $Y$ say, of $S_{2}$ in $S-R^{\prime}$. Since by hypothesis $R$ contains a point of $K, P$ say, there exists an arc $Y P$, by Condition 2 , such that $Y P-P$ is contained in $S_{2}$. But this is impossible* since $J$ does not contain a point of $S_{2}$. Therefore $S_{2}$ is contained in $R$. Assume that there

${ }^{*}$ Use is here made of the fact: a connected point set which contains a point of a domain, $D$, and a point not in $D$, contains a boundary point of $D$. 
exists a point $P$ of $K$ in $S-R^{\prime}$. Let $W$ be a point of $S_{2}$. There exists then an arc $P W$, by Condition 2, such that $P W-W$ is contained in $S_{2}$ which is impossible. Therefore $R$ contains $K-(A)$.

Lemma VII. If there exists a region $Z$, and if $K$ is such that Conditions 1 and 2 hold, then $K_{1}{ }^{\prime}$ is a connected point set.

Assume that $K_{1}{ }^{\prime}=K_{11}+K_{12}$ separate. Then, by Lemma III, there exists a simple closed curve $J_{1 i}(i=1$, and 2$)$ enclosing points of $K_{1 i}$ and containing at most $A_{1}+A_{2}$ of $K$ and such that $J_{11} \times J_{12}=0 . J_{11}$ may contain $A_{1}$, in which case $J_{12}$ will contain at most $A_{2}$ of $K$. Therefore in every possible case one of these curves contains at most one point of $K$. Say $J_{11}$ does. Then $J_{11}$, by Lemma $\mathrm{V}$, is contained, except for possibly one point, in $S_{i}(i=1$, or 2$)$ and so, by Lemma VI, encloses all except possibly one point of $K$. But it is shown in Lemma II that $K_{2}$ is non-vacuous and hence there are points of $K$ in $S-Z^{\prime}$. But $J_{11}$ is contained in $Z^{\prime}$ and so $K$ is. Therefore our assumption is incorrect and so $K_{1}{ }^{\prime}$ is a connected point set.

Lemma VIII. If there exists a region $Z$, and if $K$ is such that Conditions 1 and 2 hold, and if $K_{2}{ }^{\prime}$ is bounded, then $K_{2}{ }^{\prime}$ is a connected point set.

Assume that $K_{2}{ }^{\prime}=K_{21}+K_{22}$ separate. Then there exists a simple closed curve $J_{2 i}(i=1$, and 2$)$ enclosing points of $K_{2 i}$, contained in $S-Z$, containing at most $A_{1}+A_{2}$ of $K$, and such that $J_{21} \times J_{22}=0$, by Lemma IV. If one curve contains $A_{1}+A_{2}$ the other one does not contain a point of $K$; if one contains at most one point of $K$ the other contains at most one point of $K$. Consider, for example, the case where $J_{21}$ contains $A_{1}+A_{2}$, and let $R_{21}$ be the region bounded by this curve. Consider now how $J_{21}$ was obtained in Lemma IV. If it contains $A_{i}$ it must contain the $\operatorname{arc} C_{i 1} A_{i} C_{i 2}$ which is common to the boundaries of the region $T_{i 2}$ and the region $Z$, for only the boundaries of these two regions contained $A_{i}$ in Lemma IV. Thus there exists a simple closed curve, 
$J_{21}^{\prime \prime}$ say, which is a subset of $C$ and $J_{21}$ and which encloses $Z$ and $R_{21}$, and also encloses $T_{1}$ and $T_{2}$. Therefore $J_{21}{ }^{\prime \prime}$ does not contain $A_{i}(i=1$, or 2$)$. Thus in each possible case we obtain two curves each contained in $S_{j}(j=1$, or 2$)$ except for possibly one point, by Lemma V. Then each curve encloses all except possibly one point of $K$, by Lemma VI. Consider, for example, the case where $J_{21}$ and $J_{22}$ have this property. Since they have no points in common and since each encloses all except possibly one point of $K$, one must enclose the other. Say $J_{21}$ encloses $J_{22}$. Consider again how $J_{21}$ was obtained in Lemma IV. It is composed of the boundaries of regions each of which, among other properties, contains points of $K_{21}$. Say $N$ is one of these regions and that it contains the point $P$ of $K_{21} . N^{\prime}$ does not contain a point of $J_{22}$ for $N$ was so selected in the proof of Lemma IV. Let $R_{22}$ be the region bounded by $J_{22}$. Since $N$ has boundary points in $S-R_{22}^{\prime}$ and since it does not contain boundary points of $R_{22}$, it, and so points of $K$, are contained in $S-R_{22}^{\prime}$. Thus $J_{22}$ does not enclose all except possibly one point of $K$ on $J_{22}$. Therefore $K_{2}^{\prime}$ is a connected point set.

LEMmA IX. If there exists a region $Z$, if $K_{2}{ }^{\prime}$ is bounded, and if $K$ is such that Conditions 1 and 2 hold, then the omission of any point $P_{i}(i=1$, and 2$)$ of $K_{i}{ }^{\prime}, P_{i} \neq A_{1}$ or $A_{2}$, disconnects $K_{i}{ }^{\prime}$.

There exists an $\operatorname{arc} Q_{i 1} P_{i} Q_{i 2}$ which is, except for its end points, contained in $Z$ or in $S-Z^{\prime}$ according as $i=1$ or 2, where $Q_{i j} P_{i}-P_{j}(j=1$, or 2$)$ is contained in $S_{j}$, by Condition 2. The arc $Q_{11} P_{1} Q_{12}$, by Theorem 25 , divides $Z$ into two regions, $Z_{11}$ and $Z_{12}$ say, where $Z_{i j}^{\prime}\left(j=1\right.$, and 2) contains $A_{j}$. Let $K X\left(Z_{1 n}+A_{n}\right)=K_{1 n}$. The sets $K_{1 n}$ are non-vacuous by Lemma II. Therefore $K_{1}^{\prime}-P_{1}=K_{11}+K_{12}$ separate. Also $Q_{21} Q_{22}$ of $C$ and $Q_{21} P^{2} Q_{22}$ enclose a region, $Z^{\prime \prime}$ say. Take, for example, the case where $Q_{21} Q_{22}$ contains $A_{1}$. Let $Z^{\prime \prime} \times K_{2}$ $+A_{1}=K_{21}$ and $\left(S-Z^{\prime \prime}\right) \times K_{2}+A_{2}=K_{22}$. Then $K_{2}^{\prime}-P_{2}$ $=K_{21}+K_{22}$ separate. 
LEMмA X. If there exists a region $Z$, and if $K$ is bounded and such that Conditions 1 and 2 hold, then $K_{i}{ }^{\prime}(i=1$, or 2) is a simple continuous arc.

$K_{i}{ }^{\prime}$ is bounded and closed by hypothesis, connected by Lemma VII and Lemma VIII, and disconnected by the omission of any point other than $A_{1}$ or $A_{2}$, by Lemma IX. Therefore, by Definition $\mathrm{A}, K_{i}{ }^{\prime}$ is a simple continuous arc with end points $A_{1}$ and $A_{2}$.

Theorem I. This is Theorem A with Condition 3 omitted.

By Lemma I, there exist two points of $K$. Then, by Lemma II, there exists a region $Z$. Thus, by Lemma $\mathrm{X}, K$ is the sum of two simple continuous arcs having only their end points in common. Therefore, by Definition $\mathrm{B}, K$ is a simple closed curve.

Lemma XI. If $K$ is such that Condition 1 holds, and if $S_{i}$ ( $i=1$, and 2) is unbounded, then $K$ is not a simple closed curve.

Assume that $K$ is a simple closed curve, enclosing the region $R$. There exists a point of $S_{1}$ and a point of $S_{2}$ in $S-R^{\prime}$, for $S_{i}(i=1$, and 2$)$ is unbounded. Then there exists an arc joining these two points and contained in $S-R^{\prime}$. This arc does not contain a point of $K$, contrary to hypothesis. Therefore $K$ is not a simple closed curve.

Theorem II. This is the same as Theorem B with Condition 3 omitted.

There exist at least two distinct points of $K$ by Lemma I, $P_{1}$ and $P_{2}$ say. Assume that $K=N_{1}+N_{2}$ separate and let $A_{i}(i=1$, and 2$)$ be a point of $N_{i}$. There exists a region $Z$ by Lemma II. Then, by Lemma VII, there exists a connected subset of $K$, contained in $Z^{\prime}$, and containing $A_{1}+A_{2}$. But that this connected subset of $K$ should contain points of both of the separate point sets $N_{1}$ and $N_{2}$ is impossible. Therefore our assumption is incorrect and so $K$ is a connected point set. 
$K$ is disconnected by the omission of any two points, $A_{1}$ and $A_{2}$. For there exists a region $Z$, by Lemma II, having these two points and only these two points of $K$ on its boundary and such that $Z$ and $S-Z^{\prime}$ each contain points of $K$. Thus the omission of these two points divides $K$ into two separate point sets $K \times Z$ and $K \times\left(S-Z^{\prime}\right) . \quad K$ is a closed point set by hypothesis. Thus by Definition $C$, if $K$ consists entirely of non-cut points, $K$ is a simple closed curve, contrary to Lemma XI. Thus there exists a cut point, $P$ say, of $K$. Let $K-P=M_{1}+M_{2}$.

Let $A_{i}(i=1$, and 2$)$ be any point of $M_{i}$. Then there exists a region $Z$ by Lemma II, which, by Lemma VII, contains a connected subset of $K$ joining $A_{1}$ and $A_{2}$. That is, this connected point set joins points of the two separate point sets $M_{1}$ and $M_{2}$. This is impossible unless it contains $P$. Just as in Lemma X, $K_{1}{ }^{\prime}$ being bounded here is an arc. Both $M_{1}$ and $M_{2}$ have points in $Z$ as $P$ is clearly a limit point of both. Now $K_{1}{ }^{\prime}-P$ is the sum of two connected sets, each of which can lie, obviously, in only one of the sets $M_{1}, M_{2}$. Thus it is seen that one of these connected sets must be contained in $M_{1}$ and one in $M_{2}$. Let $M_{i} \times\left(Z+A_{i}\right)=M_{i 1}$ and $M_{i} \times\left(S-Z^{\prime}\right)=M_{i 2}$. These point sets are non-vacuous by Lemma II.

The sets $M_{12}$ and $M_{2}$ are separate since $M_{1}$ and $M_{2}$ are separate. Also $\left(M_{11}-A_{1}\right)$ and $M_{12}$ are separate for $Z$ and $S-Z^{\prime}$ are separate. $P$ is not a limit point of $S-Z$ and so is not a limit point of $M_{12}$. Therefore $K-A_{1}=M_{12}+(P$ $\left.+\left(M_{11}-A_{1}\right)+M_{2}\right)$ separate. Therefore the omission of any point of $M_{1}$ disconnects $K$. Similarly for any point of $M_{2}$. Therefore the omission of any point of $K$ disconnects $K$.

Let $P$ be any point of $K$ and let $K-P=N_{1}+N_{2}$ separate. Assume that $N_{1}=N_{11}+N_{12}$ separate. Let $A_{i}(i=1$, and 2) be any point of $N_{1 i}$. There exists by Lemma II a region $Z$ such that $K \times Z^{\prime}=A_{1} A_{2}$ is a simple continuous arc as in Lemma $X$. This arc joins points of the two separate point sets $N_{11}$ and $N_{12}$. This is impossible unless it contains $P$. Thus this arc is the sum of two $\operatorname{arcs} P A_{1}$ and $P A_{2}$. The arc 
$P A_{1}$ cannot contain points of $N_{2}$ for it would then contain a connected point set containing points of the two separate point sets $N_{1}$ and $N_{2}$. Similarly, it cannot contain points of $N_{12}$. Therefore $P A_{1}-P$ must be contained in $N_{11}$. Similarly, $P A_{2}-P$ must be contained in $N_{12}$. Therefore the arc $A_{1} A_{2}-P$ must be contained in $N_{1}$. Therefore the region $Z$ does not contain points of $N_{2}$. Thus $P$ is not a limit point of $N_{2}$. But it is well known that $N_{2}+P$ is a connected point set and so $P$ is a limit point of $N_{2}$. Therefore our assumption is incorrect and so $N_{i}(i=1$, and 2$)$ is a connected point set.

$K$ is closed by hypothesis. Therefore $K$ is an open curve.

A simple proof that Condition 3 is unnecessary in Theorems $A$ and $B$ may also be obtained from Lemma I, Lemma II, and the following theorem.

Theorem III. If $L$ is a subset of a point set $M$, and if $M^{\prime}-L=M_{1}+M_{2}$ such that (2) every point of $M_{i}(i=1$ and 2$)$ can be joined to any point $Q$ of $L$ by a simple continuous arc $P Q$ such that $P Q-Q$ is contained in $M_{i}$, and (3) $M_{j}(j=1$, or 2) is not arc-wise connected, $L$ contains at most two points.

Take for example the case where $M_{1}$ is not arc-wise connected, there being no arc $A B$ of $M_{1}$ joining the two points $A$ and $B$ of $M_{1}$. Assume that $L$ contains at least three points, $E_{i}(i=1,2$, and 3$)$ say. Then by Condition 2 there exist three $\operatorname{arcs} A E_{i}$ and three arcs $B E_{i}$ contained in $M_{1}+$ $\left(E_{1}+E_{2}+E_{3}\right)$. Then there exist three arcs, $A_{1} E_{i}$, distinct except for $A_{1}$, contained in $A E_{1}+A E_{2}+A E_{3}$ and three arcs $B_{1} E_{i}$, distinct except for $B_{1}$, contained in $B E_{1}+B E_{2}+B E_{3}$. These two sets of three arcs have but $E_{1}+E_{2}+E_{3}$ in common for otherwise there exists an arc $A B$ in $M_{1}$. There exist then three arcs $A_{1} E_{i} B_{1}(i=1,2,3)$ having only $A_{1}$ and $B_{1}$ in common. Thus there exist three simple closed curves $A_{1} E_{1} B_{1}+A_{1} E_{2} B_{1}=J_{1}$ say, $A_{1} E_{2} B_{1}+A_{1} E_{3} B_{1}=J_{2}$ say, and $A_{1} E_{3} B_{1}+A_{1} E_{1} B_{1}=J_{3}$ say. Let $R_{i}(i=1,2$, and 3$)$ be the region bounded by $J_{i}$. Consider for example the case where $R_{1}=R_{2}+R_{3}+A_{1} E_{3} B_{1}-\left(A_{1}+B_{1}\right) . J_{1}$ is contained in $M_{1}+L$. 
Assume that $S-R_{1}{ }^{\prime}$ contains a point, say $U$, of $M_{2}$. Then by Condition 2 there exists an arc $U E_{3}$ contained in $M_{2}+E_{3}$. This is impossible for such an arc must contain a point of $J_{1}$. Therefore $M_{2}$ is contained in $R_{1}$. Similarly it can be proved that $M_{2}$ is contained in $S-R_{2}^{\prime}$ and so in $R_{3}$. Similarly it can be proved that $M_{2}$ is contained in $S-R_{3}^{\prime}$. Thus a contradiction is obtained. Therefore there do not exist three points of $L$.

Cornell University

\section{TWO CLASSES OF PERIODIC ORBITS WITH REPELLING FORCES*}

BY T. H. RAWLES

1. Introduction. The purpose of this paper is to obtain certain classes of periodic orbits $\nmid$ of a system consisting of one body of very great mass which attracts two mutually repellent bodies of very small mass. The forces of attraction and repulsion in the system are assumed to vary inversely as the squares of the distances. It will be shown that if certain conditions of symmetry are imposed the orbits of the two small bodies must lie either in two parallel planes or else be coplanar. In the former case they are circles about a line perpendicular to the two planes and passing through the large body. For the second type of orbit a development in powers of a parameter will be obtained.

2. The Equation of Motion. By a proper choice of the units of time and space we can make the coefficient of the intensity of the attraction equal to 1 . Then if $K^{2}$ is the

* Presented to the Society, February 25, 1928.

$\dagger$ The orbits referred to were first calculated by Langmuir, who employed numerical integration. (Physical Review, vol. 17 (1921), p. 339.) They have been discussed by Van Vleck in his monograph on Quantum Principles and Line Spectra. (Bulletin of the National Research Council, Number 54, p. 89.) 